hep-th/9508139

BRX-TH-378

\title{
SUPERSPACE MEASURES, INVARIANT ACTIONS, AND COMPONENT PROJECTION FORMULAE FOR $(2,2)$ SUPERGRAVITY
}

\author{
Marcus T. Grisaruf \\ and \\ Marcia E. Wehlauा \\ Physics Department, Brandeis University, Waltham, MA 02254, USA
}

\begin{abstract}
In the framework of the prepotential description of superspace twodimensional $(2,2)$ supergravity, we discuss the construction of invariant integrals. In addition to the full superspace measure, we derive the measure for chiral superspace, and obtain the explicit expressions for going from superspace actions to component actions. We consider both the minimal $U_{A}(1)$ and the extended $U_{V}(1) \otimes U_{A}(1)$ theories.
\end{abstract}

August 1995

* Work partially supported by the National Science Foundation under grant PHY-92-22318.

${ }^{\dagger}$ Current address: Mars Scientific Consulting, 28 Limeridge Dr., Kingston, ON CANADA K7K 6M3 


\section{Introduction}

Recently [1], we have described the solution of the constraints for $(2,2)$ supergravity in $(2,2)$ superspace in terms of unconstrained prepotentials. For the nonminimal $U_{V}(1) \otimes U_{A}(1)$ theory, these are a real vector superfield $H^{m}$ and a general scalar superfield $S$. In the "degauged" minimal theory where one of the $U(1)$ connections is set to zero, a further constraint expresses the superfield $S$ in terms of a chiral superfield $\sigma$ (for the $U_{A}(1)$ theory), or a twisted chiral superfield $\tilde{\sigma}$ (for the $U_{V}(1)$ theory). We gave explicit expressions for the vielbein, the connections, and the vielbein determinant $E$. With these results at hand, several applications become possible. In particular, we have studied the theory in light-cone gauge and derived the Ward identities associated with the (nonlocal) induced $(2,2)$ supergravity action [2]. One can also discuss the fully supersymmetric quantization of the theory. We have described in a separate work the general coupling of two-dimensional $(2,2)$ supersymmetric matter to supergravity and the corresponding component actions [3].

For this latter application, a knowledge of invariant superspace measures in full superspace and in chiral (or twisted chiral) superspace is necessary, as well as projection formulae that allow one to obtain the corresponding component actions. In this paper we describe this aspect of the theory - the construction of invariant actions, the determination of the measures (in particular of chiral or twisted chiral densities), and the projection formulae that enable us to go from superspace actions to component actions. In the words of an esteemed colleague "the construction of superinvariants is more an art than a science". We show here that, at least in the two-dimensional $(2,2)$ theory, this construction is in fact a science.

Our paper is organized as follows: in section 2 we summarize the relevant information about the $(2,2)$ supergravity theory and the solution of the constraints. In section 3 we show how, for the minimal $U_{A}(1)$ theory, one obtains the chiral measure for integration over chiral superspace (or, equivalently, the twisted chiral results for the $U_{V}(1)$ theory). In section 4 , we derive the component projection formula for going from full superspace (or chiral superspace) to components. In the same section we obtain an alternative projection formula which involves at an intermediate stage a twisted chiral projector rather than the chiral projector. In section 5 , we show how to express the covariant derivatives of the $U_{V}(1) \otimes U_{A}(1)$ theory in terms of those of the degauged $U_{A}(1)$ theory, and derive the chiral measure for the former. Section 6 contains our conclusions. We use the conventions of refs. [1, 2]. Appendix A contains the definition of the supergravity component fields in Wess-Zumino gauge, and additional information about these derivatives. Appendices $\mathrm{B}$ and $\mathrm{C}$ contain some details of the derivations in the main text. 


\section{The $(2,2)$ constraints and their solution}

We summarize in this section the main results of refs. [1, 2]. Tangent space Lorentz, $U_{V}(1)$ and $U_{A}(1)$ generators, denoted here by $\mathcal{M}, \mathcal{Y}$ and $\mathcal{Y}^{\prime}$ respectively, are defined by their action on spinors:

$$
\begin{aligned}
& {\left[\mathcal{M}, \psi_{ \pm}\right]= \pm \frac{1}{2} \psi_{ \pm} \quad, \quad\left[\mathcal{M}, \psi_{ \pm}\right]= \pm \frac{1}{2} \psi_{ \pm} \quad} \\
& {\left[\mathcal{Y}, \psi_{ \pm}\right]=-\frac{i}{2} \psi_{ \pm} \quad, \quad\left[\mathcal{Y}, \psi_{ \pm}\right]=+\frac{i}{2} \psi_{ \pm},} \\
& {\left[\mathcal{Y}^{\prime}, \psi_{ \pm}\right]=\mp \frac{i}{2} \psi_{ \pm} \quad, \quad\left[\mathcal{Y}^{\prime}, \psi_{ \pm}\right]= \pm \frac{i}{2} \psi_{ \pm} .}
\end{aligned}
$$

It is also useful to define the combinations

$$
\begin{aligned}
& M=\frac{1}{2}\left(\mathcal{M}+i \mathcal{Y}^{\prime}\right) \quad, \quad \bar{M}=\frac{1}{2}\left(\mathcal{M}-i \mathcal{Y}^{\prime}\right) \\
& N=\frac{1}{2}(\mathcal{M}+i \mathcal{Y}) \quad, \quad \bar{N}=\frac{1}{2}(\mathcal{M}-i \mathcal{Y}) \text {. }
\end{aligned}
$$

The covariant derivatives are defined by

$$
\begin{aligned}
\nabla_{A} & =E_{A}+\Phi_{A} \mathcal{M}+\Sigma_{A}^{\prime} \mathcal{Y}^{\prime}+\Sigma_{A} \mathcal{Y} \\
& =E_{A}+\Omega_{A} M+\Gamma_{A} \bar{M}+\Sigma_{A} \mathcal{Y} .
\end{aligned}
$$

They satisfy the constraints which define the $2 \mathrm{D}, \mathrm{N}=2 U_{V}(1) \otimes U_{A}(1)$ supergravity,

$$
\begin{array}{ll}
\left\{\nabla_{+}, \nabla_{+}\right\}=0 \quad, \quad\left\{\nabla_{-}, \nabla_{-}\right\}=0 \\
\left\{\nabla_{+}, \nabla_{\dot{+}}\right\}=i \nabla_{\ddagger}, & \left\{\nabla_{-}, \nabla_{\dot{*}}\right\}=i \nabla_{=} \\
\left\{\nabla_{+}, \nabla_{-}\right\}=-\bar{R} \bar{M} \quad, \quad\left\{\nabla_{+}, \nabla_{\dot{*}}\right\}=-\bar{F} \bar{N} .
\end{array}
$$

From the constraints follow the additional commutators

$$
\begin{aligned}
& {\left[\nabla_{+}, \nabla_{\ddagger}\right]=0 \quad, \quad\left[\nabla_{-}, \nabla_{=}\right]=0,} \\
& {\left[\nabla_{\dot{+}}, \nabla_{\ddagger}\right]=0, \quad\left[\nabla_{\dot{+}}, \nabla_{=}\right]=0,} \\
& {\left[\nabla_{+}, \nabla_{=}\right]=-\frac{i}{2} \bar{R} \nabla_{\dot{-}}-i\left(\nabla_{\dot{L}} \bar{R}\right) \bar{M}-\frac{i}{2} \bar{F} \nabla_{-}-i\left(\nabla_{-} \bar{F}\right) \bar{N},} \\
& {\left[\nabla_{\dot{+}}, \nabla_{=}\right]=\frac{i}{2} R \nabla_{-}+i\left(\nabla_{-} R\right) M+\frac{i}{2} F \nabla_{\dot{-}}+i\left(\nabla_{\dot{-}} F\right) N,} \\
& {\left[\nabla_{-}, \nabla_{\ddagger}\right]=\frac{i}{2} \bar{R} \nabla_{\dot{+}}-i\left(\nabla_{\dot{+}} \bar{R}\right) \bar{M}+\frac{i}{2} F \nabla_{+}-i\left(\nabla_{+} F\right) N,} \\
& {\left[\nabla_{\dot{-}}, \nabla_{\ddagger}\right]=-\frac{i}{2} R \nabla_{+}+i\left(\nabla_{+} R\right) M-\frac{i}{2} \bar{F} \nabla_{\dot{+}}+i\left(\nabla_{\dot{+}} \bar{F}\right) \bar{N},}
\end{aligned}
$$

and 


$$
\begin{aligned}
{\left[\nabla_{\ddagger}, \nabla_{=}\right]=} & \frac{1}{2}\left(\nabla_{+} R\right) \nabla_{-}+\frac{1}{2}\left(\nabla_{-} R\right) \nabla_{+}-\frac{1}{2}\left(\nabla_{\dot{+}} \bar{R}\right) \nabla_{\dot{-}}-\frac{1}{2}\left(\nabla_{\dot{R}} \bar{R}\right) \nabla_{\dot{+}} \\
& -\frac{1}{2} R \bar{R} \bar{M}-\frac{1}{2} R \bar{R} M+\left(\nabla^{2} R\right) M-\left(\bar{\nabla}^{2} \bar{R}\right) \bar{M} \\
& +\frac{1}{2}\left(\nabla_{+} F\right) \nabla_{\dot{-}}+\frac{1}{2}\left(\nabla_{\dot{L}} F\right) \nabla_{+}-\frac{1}{2}\left(\nabla_{\dot{+}} \bar{F}\right) \nabla_{-}-\frac{1}{2}\left(\nabla_{-} \bar{F}\right) \nabla_{\dot{+}} \\
& -\frac{1}{2} F \bar{F} \bar{N}-\frac{1}{2} F \bar{F} N+\left(\nabla_{+} \nabla_{\dot{-}} F\right) N-\left(\nabla_{\dot{+}} \nabla_{-} \bar{F}\right) \bar{N} .
\end{aligned}
$$

Furthermore, for the minimal supergravities one restricts the gauge group so that either $F=0$ for the $U_{A}(1)$ version, or $R=0$ for the $U_{V}(1)$ version, by "degauging", i.e. by setting either $\Sigma_{A}=0$ or $\Sigma_{A}^{\prime}=0$.

The solution of the constraints is obtained in terms of the "hat" differential operators

$$
\hat{E}_{ \pm}=e^{-H} D_{ \pm} e^{H} \quad, \quad \hat{E}_{\dot{ \pm}}=e^{H} D_{\dot{ \pm}} e^{-H}
$$

with $H=H^{m} i \partial_{m}$, where $H^{m}$ is a real vector superfield. The spinorial vielbein is expressed in terms of these operators, as well as an additional (superscale and $U(1)$ compensator) general complex superfield $S$ as

$$
\begin{array}{rll}
E_{+} \equiv e^{\bar{S}}\left(\hat{E}_{+}+A_{+}^{-} \hat{E}_{-}\right) & , & E_{-} \equiv e^{\bar{S}}\left(\hat{E}_{-}+A_{-}^{+} \hat{E}_{+}\right) \\
E_{\dot{+}} \equiv e^{S}\left(\hat{E}_{\dot{+}}+A_{\dot{+}}^{\dot{-}} \hat{E}_{\dot{-}}\right) & , & E_{\dot{-}} \equiv e^{S}\left(\hat{E}_{\dot{-}}+A_{\dot{+}}^{\dot{+}} \hat{E}_{\dot{+}}\right) .
\end{array}
$$

The vectorial vielbein is calculated from the constraints. The $A$ 's, as well as the vielbein determinant $E$ are given explicitly in ref. [1]. We emphasize that they are functions of the superfield $H^{m}$ only.

The connections were derived in ref. [1], and we list them here for convenience:

$$
\begin{aligned}
& \Omega_{+}=+e^{\bar{S}}\left(\hat{E}_{-} A_{+}^{-}-A_{+}^{-} \hat{E}_{+} A_{-}^{+}\right) \\
& \Omega_{-}=-e^{\bar{S}}\left(\hat{E}_{+} A_{-}^{+}-A_{-}^{+} \hat{E}_{-} A_{+}^{-}\right) \\
& \Sigma_{+}=-2 i e^{\bar{S}}\left(\hat{E}_{+} \bar{S}+A_{+}^{-} \hat{E}_{-} \bar{S}\right)-i e^{\bar{S}}\left(\hat{E}_{-} A_{+}^{-}-A_{+}^{-} \hat{E}_{+} A_{-}^{+}\right) \\
& \Sigma_{-}=-2 i e^{\bar{S}}\left(\hat{E}_{-} \bar{S}+A_{-}^{+} \hat{E}_{+} \bar{S}\right)-i e^{\bar{S}}\left(\hat{E}_{+} A_{-}^{+}-A_{-}^{+} \hat{E}_{-} A_{+}^{-}\right) \\
& \Gamma_{+}=+2 e^{\bar{S}}\left(\hat{E}_{+} S+A_{+}^{-} \hat{E}_{-} S\right)+2 e^{\bar{S}}\left(\hat{E}_{+} \bar{S}+A_{+}^{-} \hat{E}_{-} \bar{S}\right)+e^{\bar{S}}\left(\hat{E}_{-} A_{+}^{-}-A_{+}^{-} \hat{E}_{+} A_{-}^{+}\right) \\
& \Gamma_{-}=-2 e^{\bar{S}}\left(\hat{E}_{-} S+A_{-}^{+} \hat{E}_{+} S-2 e^{\bar{S}}\left(\hat{E}_{-} \bar{S}+A_{-}^{+} \hat{E}_{+} \bar{S}\right)-e^{\bar{S}}\left(\hat{E}_{+} A_{-}^{+}-A_{-}^{+} \hat{E}_{-} A_{+}^{-}\right)\right.
\end{aligned}
$$

The $U_{V}(1) \otimes U_{A}(1)$ theory contains in addition to the irreducible supergravity multiplet a vector multiplet. A minimal theory is obtained by setting one of the field strengths $R$ or $F$ (or the corresponding connections) to zero. In ref. [1] we worked out the implications of the additional constraint $F=0$ for the minimal $U_{A}(1)$ theory 
and found that the superfield $S$ satisfies a constraint that expresses it in terms of an arbitrary covariantly chiral superfield $\sigma$ as follows:

$$
e^{S}=e^{\sigma} \frac{\left[1 \cdot e^{\stackrel{\leftarrow}{H}}\right]^{-\frac{1}{2}}}{\left[1-A_{\dot{+}}^{\dot{\dot{ }}} A_{\dot{-}}^{\dot{+}}\right]^{\frac{1}{2}}} E^{-\frac{1}{2}} \quad, \quad e^{\bar{S}}=e^{\bar{\sigma}} \frac{\left[1 \cdot e^{-\overleftarrow{H}}\right]^{-\frac{1}{2}}}{\left[1-A_{+}^{-} A_{-}^{+}\right]^{\frac{1}{2}}} E^{-\frac{1}{2}}
$$

where $\overleftarrow{H}$ indicates that the differential operator in $H^{m} i \partial_{m}$ acts on objects to its left. The unconstrained real vector superfield $H^{m}$ and the chiral scalar superfield $\sigma$ are the prepotentials of the minimal $U_{A}(1)(2,2)$ supergravity. The mirror image $U_{V}(1)$ theory is obtained by interchanging - and - indices everywhere (and interchanging $R$ with $F$, and $M$ with $N$ ), and amounts to replacing the chiral superfield $\sigma$ by a twisted chiral superfield $\tilde{\sigma}$. In the following, we shall concentrate primarily on the minimal $U_{A}(1)$ theory.

\section{From full superspace measure to chiral super- space measure}

In full superspace, invariant actions are constructed by means of the vielbein determinant as

$$
\mathcal{S}=\int d^{2} x d^{4} \theta E^{-1} \mathcal{L}
$$

where $\mathcal{L}$ is an arbitrary scalar function of superfields. The invariance of this integral under superspace coordinate transformations can be established by standard means; see for example Superspace, sect. 5.5 citeSuperspace. In addition [4, 2], one can show that for superspace transformations under which covariantly chiral and antichiral superfields transform as

$$
\Phi \rightarrow e^{i \Lambda} \Phi \quad, \quad \bar{\Phi} \rightarrow e^{i \bar{\Lambda}} \bar{\Phi}
$$

with

$$
\begin{aligned}
& \Lambda=\Lambda^{m} i \partial_{m}+\Lambda^{\alpha} i D_{\alpha}+\Lambda^{\dot{\alpha}} i D_{\dot{\alpha}} \\
& \bar{\Lambda}=\bar{\Lambda}^{m} i \partial_{m}+\bar{\Lambda}^{\alpha} i D_{\alpha}+\bar{\Lambda}^{\dot{\alpha}} i D_{\dot{\alpha}}
\end{aligned}
$$

the prepotential $H^{a}$ must transform as

$$
e^{2 H} \rightarrow e^{i \bar{\Lambda}} e^{2 H} e^{-i \Lambda}
$$

and the vielbein determinant transforms as

$$
E^{-1} e^{\overleftarrow{H}} \rightarrow E^{-1} e^{\overleftarrow{H}} e^{i \overleftarrow{\bar{\Lambda}}}
$$


In the minimal $U_{A}(1)$ theory we show now that one can rewrite the full superspace integral as an integral over chiral superspace, with an appropriate measure $\mathcal{E}$ that we determine explicitly:

$$
\mathcal{S}=\left.\int d^{2} x d^{2} \theta \mathcal{E}^{-1} \bar{\nabla}^{2} \mathcal{L}\right|_{\bar{\theta}=0}
$$

with

$$
\mathcal{E}^{-1}=e^{-2 \sigma}\left(1 . e^{\overleftarrow{H}}\right)
$$

We proceed by deriving first an explicit expression for $\bar{\nabla}^{2}$ acting on an arbitrary scalar $L$. We start with

$$
\begin{aligned}
\bar{\nabla}^{2} L & =\nabla_{\dot{+}} \nabla_{\dot{\dot{L}}} L \\
& =\left[e^{S}\left(\hat{E}_{\dot{+}}+A_{\dot{+}} \dot{\dot{E}} \hat{\dot{E}}\right)-\frac{1}{2} \Omega_{\dot{+}}\right]\left[e^{S}\left(\hat{E}_{\dot{\sim}}+A_{\dot{\dot{ }}}^{\dot{\dot{E}}} \hat{E}_{\dot{+}}\right)\right] L .
\end{aligned}
$$

From the complex conjugate of equations (2.9), with $\Sigma_{\dot{+}}=0$, we have

$$
\Omega_{\dot{+}}=-2 e^{S}\left(\hat{E}_{\dot{+}} S+A_{\dot{+}}^{\dot{\dot{*}}} \hat{\dot{E}} S\right) .
$$

Using this we rewrite $\bar{\nabla}^{2} L$ as

$$
\begin{aligned}
& \bar{\nabla}^{2} L=\left(\hat{E}_{\dot{+}}+A_{\dot{+}} \dot{\dot{+}} \hat{E}_{\dot{-}}\right) e^{2 S}\left(\hat{E}_{\dot{-}}+A_{\dot{+}}^{\dot{+}} \hat{E}_{\dot{+}}\right) L \\
& =\left(\hat{E}_{\dot{+}}+A_{\dot{+}} \dot{\dot{E}} \hat{E}_{\dot{*}}\right)\left(\hat{E}_{\dot{-}}+A_{\dot{\dot{+}}}^{\dot{+}} \hat{E}_{\dot{+}}\right) e^{2 S} L \\
& -2\left(\hat{E}_{\dot{+}}+A_{\dot{+}} \dot{\dot{+}} \hat{E}_{\dot{-}}\right)\left(\hat{E} \cdot S+A_{\dot{\dot{+}}}^{\dot{+}} \hat{E}_{\dot{+}} S\right) e^{2 S} L \text {. }
\end{aligned}
$$

Again from the complex conjugate of equations (2.9), setting $\Sigma_{\dot{-}}=0$, we obtain

$$
2\left(\hat{E} \cdot S+A_{\dot{\dot{+}}}^{\dot{+}} \hat{E}_{\dot{+}} S\right)=-\left(\hat{E}_{\dot{+}} A_{\dot{\dot{+}}}^{\dot{\dot{+}}}-A_{\dot{\dot{+}}}^{\dot{+}} \hat{E} \cdot A_{\dot{+}}^{\dot{\dot{+}}}\right),
$$

which, when substituted into the expression for $\bar{\nabla}^{2} L$ above, gives

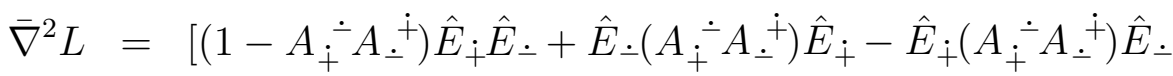

$$
\begin{aligned}
& \left.-\hat{E}_{\dot{+}} \hat{E}_{\dot{*}}\left(A_{\dot{+}}^{\dot{\dot{+}}} A_{\dot{-}}^{\dot{+}}\right)\right] e^{2 S} L \\
& =\hat{E}_{\dot{+}} \hat{E}_{\dot{-}}\left[\left(1-A_{\dot{+}}^{\dot{\dot{*}}} A_{\dot{-}}^{\dot{+}}\right) e^{2 S} L\right] \text {. }
\end{aligned}
$$

We have used the complex conjugates of (3.5) and (3.6) of ref. [1], namely,

$$
\begin{aligned}
& \hat{E}_{\dot{+}} A_{\dot{+}} \dot{\overline{\dot{ }}}+A_{\dot{+}} \dot{\bar{E}} \hat{\dot{E}} A_{\dot{+}}^{\dot{\dot{*}}}=0 \\
& \hat{E} \cdot A_{\dot{+}}^{\dot{+}}+A_{\dot{+}}^{\dot{+}} \hat{E}_{\dot{+}} A_{\dot{+}}^{\dot{+}}=0
\end{aligned}
$$

and

$$
\begin{aligned}
& \hat{E}_{\dot{+}} \hat{E}_{\dot{*}} A_{\dot{+}}^{\dot{\dot{+}}}=0 \\
& \hat{E} \cdot \hat{E}_{\dot{+}} A_{\dot{\dot{+}}}^{\dot{+}}=0 .
\end{aligned}
$$


We obtain, using (2.10)

$$
\bar{\nabla}^{2} L=\hat{E}_{\dot{+}} \hat{E} \cdot\left[e^{2 \sigma}\left(1 . e^{\overleftarrow{H}}\right)^{-1} E^{-1} L\right]
$$

We define $L^{\prime}$ by $L=e^{-2 \sigma}\left(1 . e^{\overleftarrow{H}}\right) L^{\prime}$, so that (3.15) becomes

$$
\begin{aligned}
\bar{\nabla}^{2}\left[e^{-2 \sigma}\left(1 . e^{\overleftarrow{H}}\right) L^{\prime}\right] & =\hat{E}_{\dot{+}} \hat{E}_{\dot{*}}\left(E^{-1} L^{\prime}\right) \\
& =e^{H} \bar{D}^{2} e^{-H}\left(E^{-1} L^{\prime}\right)
\end{aligned}
$$

Multiplying both sides by $e^{-H}$ and integrating over chiral superspace, we obtain

$$
\int d^{2} x d^{2} \theta \bar{D}^{2} e^{-H} E^{-1} L^{\prime}=\int d^{2} x d^{2} \theta e^{-H} e^{-2 \sigma}\left(1 . e^{\overleftarrow{H}}\right) L^{\prime}
$$

Integrating the operator $e^{-H}$ by parts on both sides of the equation gives

$$
\int d^{2} x d^{2} \theta \bar{D}^{2}\left[\left(1 . e^{\overleftarrow{H}}\right) E^{-1} L^{\prime}\right]=\int d^{2} x d^{2} \theta\left(1 . e^{\overleftarrow{H}}\right) \bar{\nabla}^{2}\left[e^{-2 \sigma}\left(1 . e^{\overleftarrow{H}}\right) L^{\prime}\right]
$$

Finally, defining $\mathcal{L}$ by $L^{\prime}=\left(1 . e^{\overleftarrow{H}}\right)^{-1} \mathcal{L}$, we obtain

$$
\int d^{2} x d^{2} \theta \bar{D}^{2}\left[E^{-1} \mathcal{L}\right]=\int d^{2} x d^{2} \theta e^{-2 \sigma}\left(1 . e^{\overleftarrow{H}}\right) \bar{\nabla}^{2} \mathcal{L}
$$

We have used the fact that $\sigma$ is covariantly chiral to pull the factor $e^{-2 \sigma}$ past the covariant derivatives. Since one can go from a full superspace integral to a $d^{2} \theta$ integral by

$$
\int d^{2} x d^{4} \theta E^{-1} \mathcal{L}=\left.\int d^{2} x d^{2} \theta \bar{D}^{2}\left[E^{-1} \mathcal{L}\right]\right|_{\bar{\theta}=0}
$$

the relation above leads us to the desired result (and determines the chiral measure $\mathcal{E})$ :

$$
\int d^{2} x d^{4} \theta E^{-1} \mathcal{L}=\left.\int d^{2} x d^{2} \theta e^{-2 \sigma}\left(1 . e^{\overleftarrow{H}}\right) \bar{\nabla}^{2} \mathcal{L}\right|_{\bar{\theta}=0}
$$

By construction, the above integral is an invariant. We can replace $\bar{\nabla}^{2} \mathcal{L}$ by any covariantly chiral expression $\mathcal{L}_{\text {chiral }}$, transforming under superspace coordinate transformations as

$$
\mathcal{L}_{\text {chiral }} \rightarrow e^{i \Lambda} \mathcal{L}_{\text {chiral }}
$$

and deduce the transformation properties of the measure

$$
e^{-2 \sigma}\left(1 \cdot e^{\overleftarrow{H}}\right) \rightarrow e^{-2 \sigma}\left(1 \cdot e^{\overleftarrow{H}}\right) e^{i \overleftarrow{\Lambda}}
$$

Obviously, a similar result holds for the decomposition to an antichiral integral.

In the $U_{V}(1)$ theory corresponding results, in terms of the twisted chiral compensator, can be obtained for twisted chiral integrals of the type $\int d^{2} x d \theta^{+} d \theta^{\dot{ }}$. 


\section{From full superspace measure to component in- tegrals}

\subsection{The chiral density projection formula}

We have shown in the previous section that it is possible to express the full superspace integral in terms of a chiral integral. In the next step of the projection we rewrite this as a component integral over ordinary $d^{2} x$ space. By dimensional arguments, and from an examination of the index structure of the possible terms, the density formula for a general $\mathcal{L}$ must take the form

$$
\begin{aligned}
\int d^{2} x d^{4} \theta E^{-1} \mathcal{L} & =\left.\int d^{2} x d^{2} \theta \mathcal{E}^{-1} \bar{\nabla}^{2} \mathcal{L}\right|_{\bar{\theta}=0} \\
& =\left.\int d^{2} x e^{-1}\left[\nabla^{2}+X^{+} \nabla_{+}+X^{-} \nabla_{-}+Y\right] \bar{\nabla}^{2} \mathcal{L}\right|_{\theta=\bar{\theta}=0}
\end{aligned}
$$

where $e$ is the ordinary space zweibein determinant and $X^{+}, X^{-}$, and $Y$ are to be determined. In the past, the determination of the coefficients $X, Y$, has been done essentially by requiring that the final component action be invariant under supersymmetry transformations. Our derivation is based on the idea that we should obtain the same result for the component action whether we go through the intermediate stage of a chiral integral, as in the equation above, or through a corresponding antichiral integral. We illustrate the procedure by using for $\mathcal{L}$ the free lagrangian for the chiral multiplet. As we shall see, proceeding from the above expression, we obtain a result which is not automatically symmetric in the auxiliary fields $F, \bar{F}$ of the chiral multiplet. Requiring that the result be symmetric leads to a unique determination of the coefficients $X, Y$.

We consider the kinetic action for the chiral multiplet

$$
\begin{aligned}
\int d^{2} x d^{4} \theta E^{-1} \bar{\Phi} \Phi= & \int d^{2} x e^{-1}\left[\nabla^{2}+X^{\alpha} \nabla_{\alpha}+Y\right] \bar{\nabla}^{2}(\bar{\Phi} \Phi) \mid \\
= & \int d^{2} x e^{-1}\left[\left(\nabla^{2} \bar{\nabla}^{2} \bar{\Phi}\right) \Phi\left|+\left(\nabla_{+} \bar{\nabla}^{2} \bar{\Phi}\right)\left(\nabla_{-} \Phi\right)\right|-\left(\nabla_{-} \bar{\nabla}^{2} \bar{\Phi}\right)\left(\nabla_{+} \Phi\right) \mid\right. \\
& +\left(\bar{\nabla}^{2} \bar{\Phi}\right)\left(\nabla^{2} \Phi\right)\left|+X^{+} \nabla_{+}\left(\bar{\nabla}^{2} \bar{\Phi} \Phi\right)\right| \\
& \left.+X^{-} \nabla_{-}\left(\bar{\nabla}^{2} \bar{\Phi} \Phi\right)\left|+Y\left(\bar{\nabla}^{2} \bar{\Phi}\right) \Phi\right|\right]
\end{aligned}
$$

according to the density formula.

The (covariant) components of the chiral multiplet are defined by

$$
\begin{array}{rll}
\Phi \mid=\phi & , \quad \bar{\Phi}=\bar{\phi} \\
\nabla_{+} \Phi \mid=\psi_{+} & , \quad \nabla_{\dot{+}} \bar{\Phi}=\psi_{\dot{+}} \\
\nabla_{-} \Phi \mid=\psi_{-} & , \quad \nabla_{\dot{\Phi}} \bar{\Phi} \mid=\psi_{\dot{\check{L}}} \\
\nabla^{2} \Phi \mid=-i F & , \quad \bar{\nabla}^{2} \bar{\Phi} \mid=-i \bar{F} .
\end{array}
$$


supergravity Appendix A. In Appendix B we present the complete expressions for the other component quantities (involving additional derivatives of $\Phi$ ) appearing above. For our present purposes, we find that the unknowns can be determined just by looking at the terms in those expressions that contain the auxiliary fields, $F, \bar{F}$. We list the relevant terms here:

$$
\begin{aligned}
\nabla_{+} \bar{\nabla}^{2} \bar{\Phi} \mid & \sim \psi_{\ddagger}^{\dot{+}} \bar{F} \\
\nabla_{-} \bar{\nabla}^{2} \bar{\Phi} \mid & \sim \psi_{=}^{\dot{\dot{F}}} \bar{F} \\
\nabla^{2} \bar{\nabla}^{2} \bar{\Phi} \mid & \sim-i\left(\psi_{\mp}^{\dot{\dot{\Phi}}} \psi_{=}^{\dot{+}} \bar{F}-\psi_{\ddagger}^{\dot{+}} \psi_{=}^{\dot{\dot{ }}} \bar{F}\right)-\frac{i}{2} \bar{B} \bar{F}
\end{aligned}
$$

where we have also used the definition $R|=B, \bar{R}|=\bar{B}$.

Substituting into (4.2), the sum of the terms containing auxiliary fields is

$$
\begin{aligned}
& i \psi_{\mp}^{\dot{+}} \psi_{=}^{\dot{亠}} \bar{F} \phi-i \psi_{\ddagger}^{\dot{\dot{ }}} \psi_{=}^{\dot{+}} \bar{F} \phi+\psi_{\mp}^{\dot{+}} \bar{F} \psi_{-}-\psi_{=}^{\dot{\dot{F}}} \bar{F} \psi_{+} \\
- & \frac{i}{2} \bar{B} \bar{F} \phi-\bar{F} F+X^{+} \psi_{\mp}^{\dot{+}} \bar{F} \phi-i X^{+} \bar{F} \psi_{+}-i X^{-} \bar{F} \psi_{-} \\
+ & X^{-} \psi_{=}^{\dot{\dot{F}}} \bar{F} \phi-i Y \bar{F} \phi .
\end{aligned}
$$

Clearly, except for the $\bar{F} F$ term, this expression is not symmetric in barred and unbarred quantities, and will not agree with the result we would obtain by going through the intermediate stage of an antichiral integral unless we set to zero the coefficients of the $\bar{F} \phi$ and the $\bar{F} \psi_{ \pm}$terms. We obtain

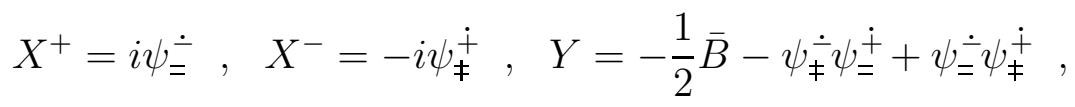

leaving $\bar{F} F$ as the only contribution. The form of the chiral density formula is thus determined.

We have, for the final result, the chiral density projection formula

$$
\begin{aligned}
& \int d^{2} x d^{4} \theta E^{-1} \mathcal{L} \\
= & \int d^{2} x e^{-1}\left[\nabla^{2}+i \psi_{=}^{-} \nabla_{+}-i \psi_{\ddagger}^{\dot{+}} \nabla_{-}+\left(-\frac{1}{2} \bar{B}-\psi_{\ddagger}^{\dot{-}} \psi_{=}^{\dot{+}}+\psi_{=}^{\dot{-}} \psi_{\ddagger}^{\dot{+}}\right)\right] \bar{\nabla}^{2} \mathcal{L} \mid .
\end{aligned}
$$

This component expression can be rewritten in terms of a twisted chiral projector as discussed in the next subsection.

\subsection{The twisted chiral density projection formula}

In the previous section we have written down the density formula for going from a full superspace integral to a component expression containing as an intermediate 
ingredient a chiral integrand $\bar{\nabla}^{2} \mathcal{L}$. In this section we will derive a similar formula involving a twisted chiral integrand $\nabla_{\dot{+}} \nabla_{-} \mathcal{L}$,

$$
\begin{aligned}
& \int d^{2} x d^{4} \theta E^{-1} \mathcal{L} \\
= & \int d^{2} x e^{-1}\left[\nabla_{\dot{-}} \nabla_{+}+i \psi_{\ddagger}^{\dot{+}} \nabla_{\dot{-}}-i \psi_{=}^{-} \nabla_{+}+\left(\psi_{\ddagger}^{\dot{+}} \psi_{=}^{-}+\psi_{\ddagger}^{-} \psi_{=}^{\dot{+}}\right)\right] \nabla_{\dot{+}} \nabla_{-} \mathcal{L} \mid .
\end{aligned}
$$

We start with the following general identity, derived straightforwardly from the commutation relations and the Bianchi identities

$$
\begin{aligned}
\nabla^{2} & \bar{\nabla}^{2}+\bar{\nabla}^{2} \nabla^{2}+\nabla_{-} \bar{\nabla}^{2} \nabla_{+}+\nabla_{\dot{-}} \nabla^{2} \nabla_{\dot{+}} \\
= & \nabla_{\ddagger} \nabla_{=}-\frac{1}{2} \nabla_{+} R \nabla_{-}+\frac{1}{2} \nabla_{\dot{+}} \bar{R} \nabla_{\dot{-}} \\
= & \frac{1}{2} \nabla_{\ddagger} \nabla_{=}+\frac{1}{2} \nabla_{=} \nabla_{\ddagger}+\frac{1}{4}\left[-\left(\nabla_{+} R\right) \nabla_{-}+\left(\nabla_{-} R\right) \nabla_{+}+\left(\nabla_{\dot{+}} \bar{R}\right) \nabla_{\dot{-}}-\left(\nabla_{\dot{-}} \bar{R}\right) \nabla_{\dot{+}}\right] \\
& -\frac{1}{2}\left[R \nabla^{2}-\bar{R} \bar{\nabla}^{2}\right] .
\end{aligned}
$$

We apply this sum of operators to an arbitrary scalar (so that certain connection terms can be dropped), inside a $d^{2} x e^{-1}$ integral (so that space-time derivatives can be dropped), and then evaluate at $\theta=0$, using some of the component expressions for covariant derivatives from Appendix A, etc.

After some lengthy manipulations, that we outline in Appendix $\mathrm{C}$, we obtain the following identity:

$$
\begin{aligned}
& \int d^{2} x e^{-1}\left\{\nabla^{2} \bar{\nabla}^{2}+\bar{\nabla}^{2} \nabla^{2}+\nabla_{-} \bar{\nabla}^{2} \nabla_{+}+\nabla_{-} \nabla^{2} \nabla_{\dot{+}}\right\} \mathcal{L} \mid \\
& =\int d^{2} x e^{-1}\left\{\left[\psi_{\neq}^{+} \psi_{=}^{-}+\psi_{\ddagger}^{-} \psi_{=}^{+}-\frac{1}{2} B\right] \nabla^{2}+\left[\psi_{\ddagger}^{\dot{+}} \psi_{=}^{\dot{\dot{ }}}+\psi_{\ddagger}^{\dot{\dot{\xi}}} \psi_{=}^{\dot{+}}+\frac{1}{2} \bar{B}\right] \bar{\nabla}^{2}\right. \\
& +\left[\psi_{\ddagger}^{+} \psi_{=}^{\dot{\dot{\Xi}}}+\psi_{\ddagger}^{\dot{\dot{\Xi}}} \psi_{=}^{+}\right] \nabla_{+} \nabla_{\dot{-}}+\left[\psi_{\ddagger}^{\dot{+}} \psi_{=}^{-}+\psi_{\ddagger}^{-} \psi_{=}^{\dot{+}}\right] \nabla_{\dot{+}} \nabla_{-} \\
& +i \psi_{\ddagger}^{+}\left[\nabla \cdot \nabla^{2}+\nabla_{-}\left(\nabla_{+} \nabla_{\dot{*}}\right)\right]-i \psi_{=}^{-}\left[\nabla_{\dot{+}} \nabla^{2}+\nabla_{+}\left(\nabla_{\dot{+}} \nabla_{-}\right)\right] \\
& \left.+i \psi_{\neq}^{\dot{+}}\left[\nabla_{-} \bar{\nabla}^{2}+\nabla_{\dot{-}}\left(\nabla_{\dot{+}} \nabla_{-}\right)\right]-i \psi_{=}^{\dot{\dot{m}}}\left[\nabla_{+} \bar{\nabla}^{2}+\nabla_{\dot{+}}\left(\nabla_{+} \nabla_{\dot{\prime}}\right)\right]\right\} \mathcal{L} \mid .
\end{aligned}
$$

On the left hand side of the equation we have the sum of projectors.

We move now certain terms from one side of the equation to the other and obtain

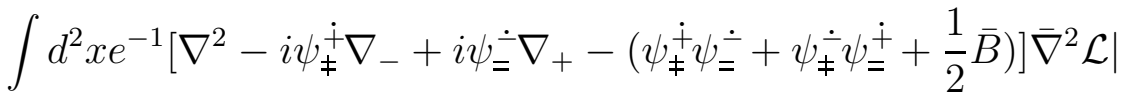

$$
\begin{aligned}
& +\int d^{2} x e^{-1}\left[\bar{\nabla}^{2}-i \psi_{\ddagger}^{+} \nabla_{\dot{-}}+i \psi_{=}^{-} \nabla_{\dot{+}}-\left(\psi_{\ddagger}^{+} \psi_{=}^{-}+\psi_{\ddagger}^{-} \psi_{=}^{+}-\frac{1}{2} B\right)\right] \nabla^{2} \mathcal{L} \mid \\
& =\int d^{2} x e^{-1}\left[\nabla_{\dot{-}} \nabla_{+}+i \psi_{\ddagger}^{\dot{+}} \nabla_{-}-i \psi_{=}^{-} \nabla_{+}+\left(\psi_{\ddagger}^{\dot{+}} \psi_{=}^{-}+\psi_{\ddagger}^{-} \psi_{=}^{\dot{+}}\right)\right] \nabla_{\dot{+}} \nabla_{-} \mathcal{L} \mid
\end{aligned}
$$

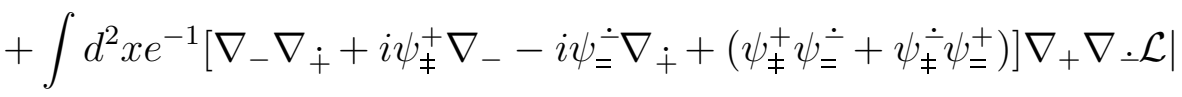


However, it can be shown that up to total derivatives the two terms on each side of the equation are equal. We obtain therefore the following result, which allows us to write a full superspace integral in terms of either chiral or twisted chiral projections,

$$
\begin{aligned}
& \int d^{2} x d^{4} \theta E^{-1} \mathcal{L} \\
& =\int d^{2} x e^{-1}\left[\nabla^{2}-i \psi_{\ddagger}^{\dot{+}} \nabla_{-}+i \psi_{=}^{\dot{\dot{\Xi}}} \nabla_{+}-\left(\psi_{\ddagger}^{\dot{+}} \psi_{=}^{\dot{\dot{=}}}+\psi_{\ddagger}^{\dot{\dot{亠}}} \psi_{=}^{\dot{+}}+\frac{1}{2} \bar{B}\right)\right] \bar{\nabla}^{2} \mathcal{L} \mid \\
& =\int d^{2} x e^{-1}\left[\nabla_{\dot{-}} \nabla_{+}+i \psi_{\ddagger}^{\dot{+}} \nabla_{\dot{-}}-i \psi_{=}^{-} \nabla_{+}+\left(\psi_{\ddagger}^{\dot{+}} \psi_{=}^{-}+\psi_{\ddagger}^{-} \psi_{=}^{\dot{+}}\right)\right] \nabla_{\dot{+}} \nabla_{-} \mathcal{L} \mid .
\end{aligned}
$$

(The asymmetry between the two forms is due to the constraint $F=0$.) Replacing $\bar{\nabla}^{2} \mathcal{L}$ or $\nabla_{\dot{+}} \nabla_{-} \mathcal{L}$ by arbitrary chiral or twisted chiral lagrangians gives the projection formulae for chiral or twisted chiral actions, respectively.

\section{From full superspace measure to chiral super- space measure in the $U_{V}(1) \otimes U_{A}(1)$ case}

In this section we derive the analogue of (3.21) for the undegauged $U_{V}(1) \otimes U_{A}(1)$ supergravity theory. We accomplish this by relating $\bar{\nabla}^{2}$ in the degauged case to $\bar{\nabla}^{2}$ in the $U_{V}(1) \otimes U_{A}(1)$ case. We consider first the covariant derivative

$$
\nabla_{\dot{+}}=E_{\dot{+}}+\Omega_{\dot{+}} \bar{M}+\Gamma_{\dot{+}} M+\Sigma_{\dot{q}} \mathcal{Y}
$$

and substitute the explicit expressions for the connections:

$$
\begin{aligned}
& \Omega_{\dot{+}}=+e^{S}\left(\hat{E} \cdot A_{\dot{+}}^{\dot{\dot{ }}}-A_{\dot{+}}^{\dot{-}} \hat{E}_{\dot{+}} A_{\dot{\dot{+}}}^{\dot{+}}\right)
\end{aligned}
$$

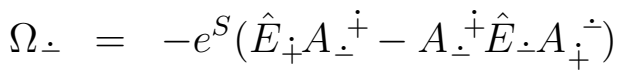

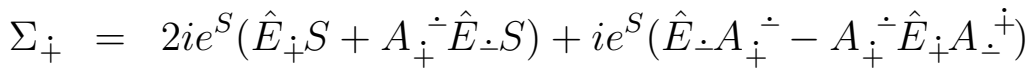

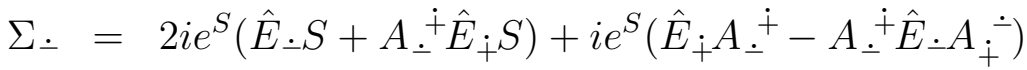

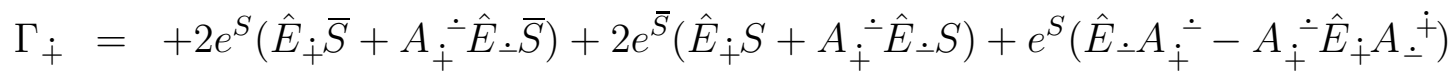

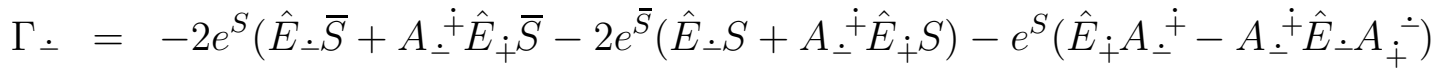

We find

$$
\nabla_{\dot{+}}=E_{\dot{+}}+\Omega_{\dot{+}}(\mathcal{M}+i \mathcal{Y})+2 E_{\dot{+}}(S+\bar{S}) M+2 i\left(E_{\dot{+}} S\right) \mathcal{Y} .
$$

We note the following identities

$$
\begin{aligned}
e^{-u \mathcal{Y}} \hat{E}_{ \pm} e^{u \mathcal{Y}} & =e^{-\frac{i}{2} u} \hat{E}_{ \pm}+e^{-\frac{i}{2} u}\left(\hat{E}_{ \pm} u\right) \mathcal{Y} \\
e^{-v M} \hat{E}_{ \pm} e^{v M} & =\hat{E}_{ \pm}+\left(\hat{E}_{ \pm} v\right) M
\end{aligned}
$$


We obtain then, from $(5.2)$

$$
\begin{aligned}
& e^{2(S+\bar{S}) M+2 i S \mathcal{Y}} \nabla_{\dot{+}} e^{-2 i S \mathcal{Y}-2(S+\bar{S}) M}=
\end{aligned}
$$

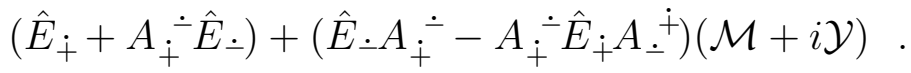

We note now that (5.4) is valid in both the undegauged and degauged theories and the right-hand-side is independent of $S$, which is the only quantity that is affected by the actual degauging. Therefore the left-hand-sides for the $U_{V}(1) \otimes U_{A}(1)$ and the $U_{A}(1)$ theories can be set equal to each other and this yields a relation between the covariant derivatives,

$$
\begin{gathered}
\left(e^{2(\mathbf{S}+\overline{\mathbf{S}}) M+2 i \mathbf{S} \mathcal{Y}} \nabla_{\dot{+}} e^{-2 i \mathbf{S} \mathcal{Y}-2(\mathbf{S}+\overline{\mathbf{S}}) M}\right)_{U_{V}(1) \otimes U_{A}(1)}= \\
\left(e^{2(S+\bar{S}) M+2 i S \mathcal{Y}} \nabla_{\dot{+}} e^{-2 i S \mathcal{Y}-2(S+\bar{S}) M}\right)_{U_{A}(1)}
\end{gathered}
$$

with a similar expression for $\nabla \dot{\sim}$. Consequently

$$
\left(\nabla_{\dot{ \pm}}\right)_{U_{V}(1) \otimes U_{A}(1)}=e^{2(S+\bar{S}-\mathbf{S}-\overline{\mathbf{S}}) M+2 i(S-\mathbf{S}) \mathcal{Y}}\left(\nabla_{\dot{ \pm}}\right)_{U_{A}(1)} e^{-2 i(S-\mathbf{S}) \mathcal{Y}-2(S+\bar{S}-\mathbf{S}-\overline{\mathbf{S}}) M}
$$

with a similar relation for the undotted derivatives. To distinguish between the two cases, we have denoted by $\mathbf{S}$ and $S$ the scale compensators in the $U_{V}(1) \otimes U_{A}(1)$ and the $U_{A}(1)$ theories, respectively (the former is a general scalar superfield whereas the latter is given by (2.10)) .

The above relation expresses the covariant derivatives of the $U_{V}(1) \otimes U_{A}(1)$ theory in terms of the covariant derivatives of the $U_{A}(1)$ theory. In particular we can write

$$
\left(\bar{\nabla}^{2}\right)_{U_{A}(1)}=e^{-2(S+\bar{S}-\mathbf{S}-\overline{\mathbf{S}}) M-2 i(S-\mathbf{S}) \mathcal{Y}}\left(\bar{\nabla}^{2}\right)_{U_{V}(1) \otimes U_{A}(1)} e^{2 i(S-\mathbf{S}) \mathcal{Y}+2(S+\bar{S}-\mathbf{S}-\overline{\mathbf{S}}) M}
$$

Acting on a scalar lagrangian $\mathcal{L}$ we can drop the exponentials following $\bar{\nabla}^{2}$, and acting on $\bar{\nabla}^{2} \mathcal{L}$ we can drop the $e^{(\cdots) M}$ term, while

$$
e^{-2 i(S-\mathbf{S}) \mathcal{Y}} \bar{\nabla}_{U \otimes U}^{2} \mathcal{L}=e^{2(S-\mathbf{S})} \bar{\nabla}_{U \otimes U}^{2} \mathcal{L}
$$

Substituting this into (3.21) we obtain

$$
\begin{aligned}
\int d^{2} x d^{4} \theta E^{-1} \mathcal{L} & =\left.\int d^{2} x d^{2} \theta e^{-2 \sigma}\left(1 . e^{\stackrel{\leftarrow}{H}}\right)\left(\bar{\nabla}^{2}\right)_{U_{A}(1)} \mathcal{L}\right|_{\bar{\theta}=0} \\
& =\left.\int d^{2} x d^{2} \theta E^{-1} \frac{e^{-2 \mathbf{S}}}{\left[1-A_{\dot{+}}^{\dot{\dot{ }}} A_{\dot{+}}^{\dot{\dot{\dot{*}}}]}\right.} \bar{\nabla}_{U \otimes U}^{2} \mathcal{L}\right|_{\bar{\theta}=0}
\end{aligned}
$$

which defines the chiral measure in the $U_{V}(1) \otimes U_{A}(1)$ theory, and allows us to construct invariant actions of the form $\int d^{2} x d^{2} \theta \mathcal{E}^{-1} \mathcal{L}_{\text {chiral }}$.

Obviously, since the theory is symmetric under the interchange of - and $\dot{-}$, a twisted chiral measure exists for writing invariants $\int d^{2} x d^{2} \tilde{\theta} \tilde{\mathcal{E}}^{-1} \mathcal{L}_{\text {twisted }}$ chiral . However, as we discuss in the concluding section, an explicit expression is harder to come by. 


\section{Discussion}

In this paper we have presented results for the integration measures in the $(2,2)$ theories, and have derived projection formulae for obtaining component actions from the corresponding superspace actions. This particular issue has been a sore point in most derivations because no clear, completely superspace technique exists in the general case. For the case at hand, at least, we have managed to avoid any reference to component supersymmetry transformations [4] or explicit $\theta$ expansions of the supergravity prepotentials in Wess-Zumino gauge [5].

Whereas in the degauged theory, which contains a chiral compensator, the existence and construction of the chiral measure is straightforward, this is not obviously the case in the undegauged theory. We have shown, by explicitly expressing the covariant derivatives of the $U_{V}(1) \otimes U_{A}(1)$ theory in terms of those of the degauged, $U_{A}(1)$ theory, how to obtain the chiral measure for the former theory, from a knowledge of the chiral measure for the latter theory.

We have also argued that, by symmetry, a twisted chiral measure exists. However, its explicit construction is not straightforward. The point is that in our solution of the constraints in ref. [1], we have chosen to express the covariant derivatives in terms of the objects $\hat{E}$ and the compensators $S$ which break the symmetry between - and - objects. To maintain the symmetry we would have to introduce additional prepotentials which were eliminated from the beginning by an appropriate $K$-transformation gauge choice.

The lack of symmetry manifests itself also in the construction of covariantly chiral and covariantly twisted chiral superfields in terms of ordinary chiral and twisted chiral ones. The construction of covariantly chiral superfields is straightforward,

$$
\Phi_{\text {cov. } \text { chiral }}=e^{H} \phi_{\text {chiral }},
$$

because then the left-hand-side is annihilated by $\nabla_{ \pm}$when the right-hand-side is annihilated by $D_{ \pm}$(c.f. (2.7)). However, if we want to obtain a covariantly twisted chiral superfield $\mathcal{X}$ satisfying

$$
\nabla_{\dot{+}} \mathcal{X}=\nabla_{-} \mathcal{X}=0
$$

in terms of an ordinary twisted chiral superfield satisfying

$$
D_{\dot{+}} \chi=D_{-} \chi=0
$$

it is clear that the above construction will not work. In fact, we have not succeeded in obtaining a closed form relation similar to (6.1). In the remainder of this concluding section we show what the relation is to first order in the supergravity prepotential. 
We begin by writing down the differential equations that the twisted chirality conditions imply for $\mathcal{X}$,

$$
\begin{aligned}
{\left[e^{-H} D_{-} e^{H}+A_{-}^{+} e^{-H} D_{+} e^{H}\right] \mathcal{X} } & =0 \\
{\left[e^{H} D_{\dot{+}} e^{-H}+A_{\dot{+}} e^{H} D_{\dot{-}} e^{-H}\right] \mathcal{X} } & =0
\end{aligned}
$$

To first order in $H$, these equations reduce to

$$
\begin{aligned}
D_{-} \mathcal{X}+i\left(D_{-} H^{a}\right) \partial_{a} \mathcal{X}+A_{-}^{+} D_{+} \mathcal{X} & =0 \\
D_{\dot{+}} \mathcal{X}-i\left(D_{\dot{+}} H^{a}\right) \partial_{a} \mathcal{X}+A_{\dot{+}}^{\dot{ }} D_{\dot{-}} \mathcal{X} & =0
\end{aligned}
$$

with

$$
\begin{aligned}
& A_{-}^{+}=-2 D_{\dot{+}} D_{-} H^{\ddagger}+\mathcal{O}\left(H^{2}\right) \\
& A_{\dot{+}}^{\dot{\bar{*}}}=-2 D_{\dot{+}} D_{-} H^{=}+\mathcal{O}\left(H^{2}\right) .
\end{aligned}
$$

We now set $\mathcal{X}=\chi+Z$, and solve iteratively for $Z$. To linear order we find that

$$
Z=-2 D_{\dot{+}} H^{\ddagger} D_{+} \chi+2 D_{-} H^{=} D_{\dot{-}} \chi+i H^{=} \partial_{=} \chi-i H^{\ddagger} \partial_{\ddagger} \chi,
$$

and therefore we can express $\mathcal{X}$ and, in a similar fashion, $\overline{\mathcal{X}}$ as

$$
\begin{aligned}
\mathcal{X} & =\left[1-2 D_{\dot{+}} H^{\ddagger} D_{+}+2 D_{-} H^{=} D_{-}+i H^{=} \partial_{=}-i H^{\ddagger} \partial_{\ddagger}\right] \chi+\mathcal{O}\left(H^{2}\right) \\
\overline{\mathcal{X}} & =\left[1+2 D_{+} H^{\ddagger} D_{\dot{+}}-2 D_{\dot{-}} H^{=} D_{-}-i H^{=} \partial_{=}+i H^{\ddagger} \partial_{\ddagger}\right] \bar{\chi}+\mathcal{O}\left(H^{2}\right) .
\end{aligned}
$$

Acknowledgments The impetus for much of this work came from our interaction with Jim Gates. We wish to thank him for discussions, suggestions, and general insights into many aspects of superspace and its geometry. We also thank Nathan Berkovits for discussions concerning the existence of chiral and twisted chiral measures in the $U_{V}(1) \otimes U_{A}(1)$ theory. M.E.W. thanks the Physics Department of Queen's University for hospitality. 


\section{Appendices}

\section{A Components of covariant derivatives}

We determine in this Appendix the components of supergravity covariant derivatives. We restrict ourselves to the minimal $U_{A}(1)$ theory by setting $\Sigma_{A}=0$. In defining components we follow the philosophy and methods described in Superspace, section 5.6 [4]. By definition of Wess-Zumino gauge, the expressions for the covariant derivatives evaluated at $\theta=0$ are:

$$
\begin{aligned}
\nabla_{\alpha} \mid & =\partial_{\alpha} \\
\nabla_{a} \mid & =\mathbf{D}_{a}+\psi_{a}^{\alpha} \nabla_{\alpha}\left|+\psi_{a}^{\dot{\alpha}} \nabla_{\dot{\alpha}}\right| \\
& =\mathbf{D}_{a}+\psi_{a}^{\alpha} \partial_{\alpha}+\psi_{a}^{\dot{\alpha}} \partial_{\dot{\alpha}}
\end{aligned}
$$

These expressions also serve to define the gravitino fields.

The component connections are defined by projection:

$$
\begin{aligned}
\Phi_{A} \mid & =\varphi_{A} \\
\Sigma_{A}^{\prime} \mid & =A_{A}^{\prime} \\
\Sigma_{A} \mid & =V_{A}
\end{aligned}
$$

and

$$
\begin{aligned}
\Omega_{A} \mid & =\omega_{A} \\
\Gamma_{A} \mid & =\gamma_{A} .
\end{aligned}
$$

Note that

$$
\varphi_{A}=\frac{1}{2}\left(\omega_{A}+\gamma_{A}\right) \text { and } A_{A}^{\prime}=\frac{i}{2}\left(\omega_{A}-\gamma_{A}\right) .
$$

In the $U_{A}(1)$ theory we define $\mathbf{D}_{a}$ as the fully covariant gravitational derivative with a Lorentz connection $\varphi_{A}$ that includes, in addition to the ordinary connection, extra terms that are bilinear in the gravitini $\psi_{a}^{\alpha}, \psi_{a}^{\dot{\alpha}}$. Specifically, $\mathbf{D}_{a}$ is defined to be

$$
\begin{aligned}
\mathbf{D}_{a} & =e_{a}+\varphi_{a} \mathcal{M}+A_{a}^{\prime} \mathcal{Y}^{\prime} \\
& =e_{a}+\omega_{a} M+\gamma_{a} \bar{M}
\end{aligned} .
$$

We also introduce the ordinary gravitational covariant derivative (without $U(1)$ connection or gravitino bilinears), denoted by $\mathcal{D}_{a}$

$$
\mathcal{D}_{a}=e_{a}+\varpi_{a} \mathcal{M} .
$$


We also need expressions for the higher $\theta$ components of the covariant derivatives. The $\theta^{\alpha}$ component of $\nabla_{\beta}$ is defined by (c.f. Superspace sec. 5.6.b)

$$
\nabla_{\alpha} \nabla_{\beta}\left|=\frac{1}{2}\left\{\nabla_{\alpha}, \nabla_{\beta}\right\}\right|
$$

while the $\theta^{\alpha}$ component of $\nabla_{b}$ is

$$
\begin{aligned}
\nabla_{\alpha} \nabla_{b} \mid & =\left[\nabla_{\alpha}, \nabla_{b}\right]\left|+\nabla_{b} \nabla_{\alpha}\right| \\
& =\left[\nabla_{\alpha}, \nabla_{b}\right]\left|+\mathbf{D}_{b} \nabla_{\alpha}\right|+\psi_{b}^{\beta} \partial_{\beta} \nabla_{\alpha}\left|+\psi_{b}^{\dot{\beta}} \partial_{\dot{\beta}} \nabla_{\alpha}\right|
\end{aligned}
$$

From (A.7) and (2.3) we obtain the following results:

$$
\begin{aligned}
& \nabla_{+} \nabla_{+} \mid=\frac{1}{2}\left\{\nabla_{+}, \nabla_{+}\right\}=0 \\
& \nabla_{-} \nabla_{-} \mid=\frac{1}{2}\left\{\nabla_{-}, \nabla_{-}\right\}=0 \\
& \nabla_{+} \nabla_{\dot{+}} \mid=\frac{1}{2}\left\{\nabla_{+}, \nabla_{\dot{+}}\right\}=\frac{i}{2} \nabla_{\ddagger} \\
& \nabla_{-} \nabla_{\dot{+}} \mid=\frac{1}{2}\left\{\nabla_{-}, \nabla_{\dot{-}}\right\}=\frac{i}{2} \nabla_{=} \\
& \nabla_{+} \nabla_{-}\left|=\frac{1}{2}\left\{\nabla_{+}, \nabla_{-}\right\}=-\frac{1}{2} \bar{R}\right| \bar{M} \\
& \nabla_{+} \nabla_{\dot{*}} \mid=\frac{1}{2}\left\{\nabla_{+}, \nabla_{\dot{-}}\right\}=0 \\
& \nabla_{-} \nabla_{\dot{+}} \mid=\frac{1}{2}\left\{\nabla_{-}, \nabla_{\dot{+}}\right\}=0,
\end{aligned}
$$

and from (A.8), we derive the series of identities that appears below:

$$
\begin{aligned}
& \nabla_{+} \nabla_{\ddagger}\left|=\left[\nabla_{+}, \nabla_{\ddagger}\right]\right|+\nabla_{\ddagger} \nabla_{+} \mid \\
& =\mathbf{D}_{\ddagger} \nabla_{+}\left|+\psi_{\ddagger}^{-} \nabla_{-} \nabla_{+}\right|+\psi_{\ddagger}^{\dot{+}} \nabla_{\dot{+}} \nabla_{+} \mid \\
& =\mathbf{D}_{\ddagger} \nabla_{+}\left|-\frac{1}{2} \psi_{\ddagger}^{-} \bar{R}\right| \bar{M}+\frac{i}{2} \psi_{\ddagger}^{\dot{+}}\left(\mathbf{D}_{\ddagger}+\psi_{\ddagger}^{\alpha} \nabla_{\alpha}+\psi_{\ddagger}^{\dot{\alpha}} \nabla_{\dot{\alpha}}\right) \mid \\
& \nabla_{-} \nabla_{\ddagger}\left|=\left[\nabla_{-}, \nabla_{\ddagger}\right]\right|+\nabla_{\ddagger} \nabla_{-} \mid \\
& =\mathbf{D}_{\ddagger} \nabla_{-}\left|+\psi_{\ddagger}^{+} \nabla_{+} \nabla_{-}\right|+\psi_{\ddagger}^{\dot{\bullet}} \nabla_{\dot{-}} \nabla_{-}\left|+\frac{i}{2} \bar{R} \nabla_{\dot{+}}\right|-i\left(\nabla_{\dot{+}} \bar{R}\right) \bar{M} \mid \\
& =\mathbf{D}_{\ddagger} \nabla_{-}\left|-\frac{1}{2} \psi_{\ddagger}^{+} \bar{R}\right| \bar{M}+\frac{i}{2} \psi_{\ddagger}^{\dot{\dot{\dot{S}}}}\left(\mathbf{D}_{=}+\psi_{=}^{\alpha} \nabla_{\alpha}+\psi_{=}^{\dot{\alpha}} \nabla_{\dot{\alpha}}\right)\left|+\frac{i}{2} \bar{R} \nabla_{\dot{+}}\right|-i\left(\nabla_{\dot{+}} \bar{R}\right) \bar{M} \mid \\
& \nabla_{\dot{+}} \nabla_{\ddagger}\left|=\mathbf{D}_{\ddagger} \nabla_{\dot{+}}\right|+\frac{1}{2} \psi_{\ddagger}^{\dot{\dot{亠}}} R\left|M+\frac{i}{2} \psi_{\ddagger}^{+}\left(\mathbf{D}_{\ddagger}+\psi_{\ddagger}^{\alpha} \nabla_{\alpha}+\psi_{\ddagger}^{\dot{\alpha}} \nabla_{\dot{\alpha}}\right)\right| \\
& \nabla \cdot \nabla_{\ddagger}\left|=\mathbf{D}_{\ddagger} \nabla_{\dot{ }}\right|+\frac{1}{2} \psi_{\ddagger}^{\dot{+}} R\left|M+\frac{i}{2} \psi_{\ddagger}^{-}\left(\mathbf{D}_{=}+\psi_{=}^{\alpha} \nabla_{\alpha}+\psi_{=}^{\dot{\alpha}} \nabla_{\dot{\alpha}}\right)\right|-\frac{i}{2} R \nabla_{+}\left|+i\left(\nabla_{+} R\right) M\right|
\end{aligned}
$$




$$
\begin{aligned}
& \nabla_{+} \nabla_{=}\left|=\mathbf{D}_{=} \nabla_{+}\right|-\frac{1}{2} \psi_{=}^{-} \bar{R}\left|\bar{M}+\frac{i}{2} \psi_{=}^{\dot{+}}\left(\mathbf{D}_{\ddagger}+\psi_{\ddagger}^{\alpha} \nabla_{\alpha}+\psi_{\ddagger}^{\dot{\alpha}} \nabla_{\dot{\alpha}}\right)\right|-\frac{i}{2} \bar{R} \nabla_{-}|-i(\nabla \cdot \bar{R}) \bar{M}| \\
& \nabla_{-} \nabla_{=}\left|=\mathbf{D}_{=} \nabla_{-}\right|-\frac{1}{2} \psi_{=}^{+} \bar{R}\left|\bar{M}+\frac{i}{2} \psi_{=}^{\dot{\prime}}\left(\mathbf{D}_{=}+\psi_{=}^{\alpha} \nabla_{\alpha}+\psi_{=}^{\dot{\alpha}} \nabla_{\dot{\alpha}}\right)\right| \\
& \nabla_{\dot{+}} \nabla_{=}\left|=\mathbf{D}_{=} \nabla_{\dot{+}}\right|+\frac{1}{2} \psi_{=}^{\dot{\dot{\prime}}} R\left|M+\frac{i}{2} \psi_{=}^{+}\left(\mathbf{D}_{\ddagger}+\psi_{\ddagger}^{\alpha} \nabla_{\alpha}+\psi_{\ddagger}^{\dot{\alpha}} \nabla_{\dot{\alpha}}\right)\right|+\frac{i}{2} R \nabla_{-}\left|+i\left(\nabla_{-} R\right) M\right| \\
& \nabla \cdot \nabla_{=}\left|=\mathbf{D}_{=} \nabla \cdot\right|+\frac{1}{2} \psi_{=}^{\dot{\dagger}} R\left|M+\frac{i}{2} \psi_{=}^{-}\left(\mathbf{D}_{=}+\psi_{=}^{\alpha} \nabla_{\alpha}+\psi_{=}^{\dot{\alpha}} \nabla_{\dot{\alpha}}\right)\right|
\end{aligned}
$$

where we have used the commutation relations

$$
\begin{aligned}
& {\left[\nabla_{+}, \nabla_{\ddagger}\right]=0, \quad\left[\nabla_{-}, \nabla_{=}\right]=0} \\
& {\left[\nabla_{\dot{+}}, \nabla_{\ddagger}\right]=0, \quad\left[\nabla_{\dot{\bullet}}, \nabla_{-}\right]=0} \\
& {\left[\nabla_{+}, \nabla_{\mp}\right]=-\frac{i}{2} \bar{R} \nabla_{\dot{-}}-i\left(\nabla_{\dot{-}} \bar{R}\right) \bar{M}} \\
& {\left[\nabla_{\dot{+}}, \nabla_{\mp}\right]=\frac{i}{2} R \nabla_{-}+i\left(\nabla_{-} R\right) M} \\
& {\left[\nabla_{-}, \nabla_{\ddagger}\right]=\frac{i}{2} \bar{R} \nabla_{\dot{+}}-i\left(\nabla_{\dot{+}} \bar{R}\right) \bar{M}} \\
& {\left[\nabla_{\dot{-}}, \nabla_{\ddagger}\right]=-\frac{i}{2} R \nabla_{+}+i\left(\nabla_{+} R\right) M}
\end{aligned}
$$

and also

$$
\begin{aligned}
{\left[\nabla_{\ddagger}, \nabla_{\mathbf{z}}\right]=} & \frac{1}{2}\left(\nabla_{+} R\right) \nabla_{-}+\frac{1}{2}\left(\nabla_{-} R\right) \nabla_{+}-\frac{1}{2}\left(\nabla_{\dot{+}} \bar{R}\right) \nabla_{\dot{-}}-\frac{1}{2}(\nabla \cdot \bar{R}) \nabla_{\dot{+}} \\
& -\frac{1}{2} R \bar{R} \bar{M}-\frac{1}{2} R \bar{R} M+\left(\nabla^{2} R\right) M-\left(\bar{\nabla}^{2} \bar{R}\right) \bar{M} .
\end{aligned}
$$

We also observe that

$$
\begin{aligned}
\nabla_{\ddagger} \nabla_{=} \mid & =\left(\mathbf{D}_{\ddagger}+\psi_{\ddagger}^{\alpha} \nabla_{\alpha}+\psi_{\ddagger}^{\dot{\alpha}} \nabla_{\dot{\alpha}}\right) \nabla_{=} \mid \\
& =\nabla_{\ddagger}\left|\nabla_{=}\right|+\psi_{\ddagger}^{\alpha} \nabla_{\alpha} \nabla_{=}\left|+\psi_{\ddagger}^{\dot{\alpha}} \nabla_{\dot{\alpha}} \nabla_{=}\right|,
\end{aligned}
$$

so that

$$
\left[\nabla_{\ddagger}, \nabla_{=}\right]\left|=\left[\nabla_{\ddagger}\left|, \nabla_{=}\right|\right]+\psi_{\ddagger}^{\alpha} \nabla_{\alpha} \nabla_{=}\right|+\psi_{\ddagger}^{\dot{\alpha}} \nabla_{\dot{\alpha}} \nabla_{=}\left|-\psi_{=}^{\alpha} \nabla_{\alpha} \nabla_{\ddagger}\right|-\psi_{=}^{\dot{\alpha}} \nabla_{\dot{\alpha}} \nabla_{\ddagger} \mid .
$$

The first term can be expanded as

$$
\begin{aligned}
& {\left[\nabla_{\ddagger}\left|, \nabla_{=}\right|\right]=\left[\mathbf{D}_{\ddagger}+\psi_{\ddagger}^{\alpha} \partial_{\alpha}+\psi_{\ddagger}^{\dot{\alpha}} \partial_{\dot{\alpha}}, \mathbf{D}_{=}+\psi_{\equiv}^{\beta} \partial_{\beta}+\psi_{=}^{\dot{\beta}} \partial_{\dot{\beta}}\right]} \\
& =\left[\mathbf{D}_{\ddagger}, \mathbf{D}_{=}\right]+\mathbf{D}_{[\ddagger}\left(\psi_{=]}^{\alpha} \partial_{\alpha}\right)+\mathbf{D}_{[\ddagger}\left(\psi_{=]}^{\dot{\alpha}} \partial_{\dot{\alpha}}\right) \\
& =\left[\mathbf{D}_{\ddagger}, \mathbf{D}_{=}\right]+\left(\mathbf{D}_{[\ddagger} \psi_{=]}^{\alpha}\right) \partial_{\alpha}+\left(\mathbf{D}_{[\ddagger} \psi_{=]}^{\dot{\alpha}}\right) \partial_{\dot{\alpha}} \\
& +\frac{1}{2} \omega_{[\mp}\left(\psi_{=]}^{+} \partial_{+}-\psi_{=]}^{-} \partial_{-}\right)+\frac{1}{2} \gamma_{[\neq}\left(\psi_{=]}^{\dot{+}} \partial_{\dot{+}}-\psi_{=]}^{\dot{\dot{m}}} \partial_{\dot{-}}\right) .
\end{aligned}
$$


Substituting this into $\left[\nabla_{\mp}, \nabla_{=}\right] \mid$, we get

$$
\begin{aligned}
& {\left[\nabla_{\ddagger}, \nabla_{=}\right] \mid=\left[\mathbf{D}_{\ddagger}, \mathbf{D}_{=}\right]+\mathbf{D}_{[\ddagger}\left(\psi_{=]}^{\alpha} \partial_{\alpha}\right)+\mathbf{D}_{[\ddagger}\left(\psi_{=]}^{\dot{\alpha}} \partial_{\dot{\alpha}}\right)} \\
& +\psi_{\ddagger}^{\alpha} \nabla_{\alpha} \nabla_{=}\left|+\psi_{\ddagger}^{\dot{\alpha}} \nabla_{\dot{\alpha}} \nabla_{=}\right|-\psi_{=}^{\alpha} \nabla_{\alpha} \nabla_{\ddagger}\left|-\psi_{=}^{\dot{\alpha}} \nabla_{\dot{\alpha}} \nabla_{\ddagger}\right| \\
& =\left[\mathbf{D}_{\ddagger}, \mathbf{D}_{=}\right]+\mathbf{D}_{[\ddagger}\left(\psi_{=]}^{\alpha} \partial_{\alpha}\right)+\mathbf{D}_{[\ddagger}\left(\psi_{=]}^{\dot{\alpha}} \partial_{\dot{\alpha}}\right) \\
& +\psi_{\ddagger}^{+}\left[\nabla=\partial_{+}+\frac{i}{2} \psi_{=}^{\dot{+}}\left(\mathbf{D}_{\ddagger}+\psi_{\ddagger}^{\alpha} \partial_{\alpha}+\psi_{\ddagger}^{\dot{\alpha}} \partial_{\dot{\alpha}}\right)-\frac{1}{2} \psi_{=}^{-} \bar{R} \bar{M}-\frac{i}{2} \bar{R} \partial_{-}-i(\nabla \cdot \bar{R}) \bar{M}\right] \\
& +\psi_{\ddagger}^{-}\left[\mathbf{D}_{=} \partial_{-}-\frac{1}{2} \psi_{=}^{+} \bar{R} \bar{M}+\frac{i}{2} \psi_{=}^{\dot{\doteq}}\left(\mathbf{D}_{=}+\psi_{=}^{\alpha} \partial_{\alpha}+\psi_{=}^{\dot{\alpha}} \partial_{\dot{\alpha}}\right)\right] \\
& +6 \text { other similar terms . }
\end{aligned}
$$

If we compare this now with (A.12) (which is true in general, not just at $\theta=0$ ), we can obtain expressions for the various derivatives of $R$ and $\bar{R}$, evaluated at $\theta=0$. By comparing the coefficients of $\nabla_{+} \mid=\partial_{+}$on both sides, for example, we get

$$
\frac{1}{2} \nabla_{-} R\left|=\mathbf{D}_{[\ddagger} \psi_{=]}^{+}+\frac{i}{2} \psi_{=}^{\dot{\dot{\Xi}}} R\right|+i \psi_{\ddagger}^{-} \psi_{=}^{\dot{\dot{=}}} \psi_{=}^{+}+i \psi_{\ddagger}^{\dot{+}} \psi_{=}^{+} \psi_{\mp}^{+}+i \psi_{\ddagger}^{\dot{\dot{\Xi}}} \psi_{=}^{-} \psi_{=}^{+} .
$$

In the same fashion

$$
\begin{aligned}
& \frac{1}{2} \nabla_{+} R\left|=\mathbf{D}_{[\ddagger} \psi_{=]}^{-}+\frac{i}{2} \psi_{\ddagger}^{\dot{+}} R\right|+i \psi_{\ddagger}^{+} \psi_{=}^{\dot{+}} \psi_{\ddagger}^{-}+i \psi_{\ddagger}^{-} \psi_{=}^{\dot{\dot{=}}} \psi_{=}^{-}+i \psi_{\ddagger}^{\dot{+}} \psi_{=}^{+} \psi_{\ddagger}^{-}
\end{aligned}
$$

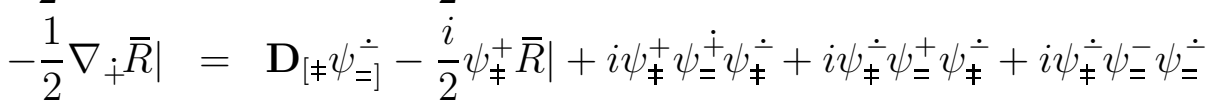

$$
\begin{aligned}
& -\frac{1}{2} \nabla \cdot \bar{R}\left|=\mathbf{D}_{[\ddagger} \psi_{=]}^{\dot{+}}-\frac{i}{2} \psi_{=}^{-} \bar{R}\right|+i \psi_{\ddagger}^{+} \psi_{=}^{\dot{+}} \psi_{\ddagger}^{\dot{+}}+i \psi_{\ddagger}^{\dot{\dot{ }}} \psi_{=}^{-} \psi_{=}^{\dot{+}}+i \psi_{\ddagger}^{-} \psi_{=}^{\dot{\dot{ }}} \psi_{=}^{\dot{+}} \\
& \left(-\frac{1}{2} R \bar{R}+\nabla^{2} R\right)\left|=\left[\mathbf{D}_{\ddagger}, \mathbf{D}_{=}\right]_{M}-i \psi_{=}^{\dot{\dot{\Xi}}} \nabla_{+} R\right|+i \psi_{\ddagger}^{\dot{+}} \nabla_{-} R\left|-\psi_{=}^{\dot{\dot{\dot{y}}}} \psi_{\ddagger}^{\dot{+}} R\right|-\psi_{=}^{\dot{+}} \psi_{\ddagger}^{\dot{\dot{亠}}} R \mid \\
& \left(-\frac{1}{2} R \bar{R}-\bar{\nabla}^{2} \bar{R}\right)\left|=\left[\mathbf{D}_{\ddagger}, \mathbf{D}_{=}\right]_{\bar{M}}-i \psi_{\ddagger}^{+} \nabla \cdot \bar{R}\right|+i \psi_{=}^{-} \nabla_{\dot{+}} \bar{R}\left|-\psi_{\ddagger}^{+} \psi_{=}^{-} \bar{R}\right|+\psi_{=}^{+} \psi_{\ddagger}^{-} \bar{R} \mid
\end{aligned}
$$

We define the "component" torsion and curvature by

$$
\left[\mathbf{D}_{\ddagger}, \mathbf{D}_{=}\right]=t_{\neq=}^{a} \mathbf{D}_{a}+r_{\neq=} M+\bar{r}_{\neq=} \bar{M}
$$

Substituting (A.5) into (A.19) we have

$$
\begin{aligned}
{\left[\mathbf{D}_{\ddagger}, \mathbf{D}_{=}\right]=} & {\left[e_{\ddagger}, e_{=}\right]+e_{[\ddagger} \omega_{=]} M+e_{[\ddagger} \gamma_{=]} \bar{M} } \\
& -\frac{1}{2} \omega_{\{=} e_{\ddagger\}}-\frac{1}{2} \gamma_{\{\ddagger} e_{=\}}-\omega_{\ddagger} \omega_{=} M \\
& +\gamma_{\ddagger} \gamma_{=} \bar{M}-\frac{1}{2} \gamma_{\{\neq} \omega_{=\}} M-\frac{1}{2} \omega_{\{=} \gamma_{\ddagger\}} \bar{M} .
\end{aligned}
$$


The anholonomy coefficients are defined as usual by $\left[e_{\ddagger}, e_{=}\right]=C_{\ddagger=}{ }^{a} e_{a}$. Comparing (A.19) with (A.20), we find the torsions

$$
\begin{aligned}
& t_{\ddagger=}^{\ddagger}=C_{\ddagger=}{ }^{\ddagger}-\frac{1}{2}(\omega+\gamma)_{=} \\
& t_{\neq=}==C_{\neq=}=-\frac{1}{2}(\omega+\gamma)_{\ddagger} .
\end{aligned}
$$

and the curvatures

$$
\begin{aligned}
& r_{\neq=}=e_{[\ddagger} \omega_{=]}-C_{\ddagger=}{ }^{\ddagger} \omega_{\ddagger}-C_{\ddagger=}={ }^{\circ} \omega= \\
& \bar{r}_{\neq=}=e_{[\ddagger} \gamma_{=]}-C_{\ddagger=}{ }^{\ddagger} \gamma_{\ddagger}-C_{\neq=}={ }^{=} \gamma_{=} .
\end{aligned}
$$

The full superspace torsions are defined in the standard way, $\left[\nabla_{A}, \nabla_{B}\right\}=T_{A B}{ }^{C} \nabla_{C}+$ $R_{A B} M+\bar{R}_{A B} \bar{M}$. Therefore, from (A.16) at $\theta=0$ and using (A.1), we obtain

$$
\begin{aligned}
& T_{\ddagger=}{ }^{\ddagger} \mid=t_{\ddagger=}{ }^{\ddagger}+i\left(\psi_{\ddagger}^{+} \psi_{=}^{\dot{+}}+\psi_{\ddagger}^{\dot{+}} \psi_{=}^{+}\right) \\
& T_{\neq=}=\mid=t_{\ddagger=}=+i\left(\psi_{\ddagger}^{-} \psi_{=}^{\dot{\bar{\Xi}}}+\psi_{\ddagger}^{\dot{\dot{亠}}} \psi_{=}^{-}\right) \\
& T_{\ddagger=}^{+}\left|=\mathbf{D}_{[\ddagger} \psi_{=]}^{+}+\frac{i}{2} \psi_{\equiv}^{\dot{\dot{\doteq}}} R\right|+i \psi_{\ddagger}^{-} \psi_{\equiv}^{\dot{\dot{\Xi}}} \psi_{=}^{+}+i \psi_{\ddagger}^{\dot{+}} \psi_{=}^{+} \psi_{\ddagger}^{+}+i \psi_{\ddagger}^{\dot{\dot{亠}}} \psi_{=}^{-} \psi_{=}^{+} \\
& T_{\ddagger=}^{-}\left|=\mathbf{D}_{[\ddagger} \psi_{=]}^{-}+\frac{i}{2} \psi_{\ddagger}^{\dot{+}} R\right|+i \psi_{\ddagger}^{+} \psi_{=}^{\dot{+}} \psi_{\ddagger}^{-}+i \psi_{\ddagger}^{-} \psi_{=}^{\dot{\dot{\Xi}}} \psi_{\equiv}^{-}+i \psi_{\ddagger}^{\dot{+}} \psi_{=}^{+} \psi_{\ddagger}^{-} .
\end{aligned}
$$

However, from (A.12), $T_{\neq=}{ }^{a}=0$. Combining this with (A.21) and (A.23), we obtain

$$
\begin{aligned}
\varphi_{\ddagger} & =\frac{1}{2}(\omega+\gamma)_{\ddagger} \\
& =C_{\ddagger=}=i\left(\psi_{\ddagger}^{-} \psi_{=}^{\dot{-}}+\psi_{\ddagger}^{\dot{亠}} \psi_{=}^{-}\right) \\
& =\varpi_{\ddagger}+i\left(\psi_{\ddagger}^{-} \psi_{=}^{\dot{亠}}+\psi_{\ddagger}^{\dot{ }} \psi_{=}^{-}\right) \\
\varphi_{=} & =\frac{1}{2}(\omega+\gamma)_{=} \\
& =C_{\ddagger=}{ }^{\ddagger}+i\left(\psi_{\ddagger}^{+} \psi_{=}^{\dot{+}}+\psi_{\ddagger}^{\dot{+}} \psi_{=}^{+}\right) \\
& =\varpi_{=}+i\left(\psi_{\ddagger}^{+} \psi_{=}^{\dot{+}}+\psi_{\ddagger}^{+} \psi_{=}^{+}\right)
\end{aligned}
$$

for the full component Lorentz connection, written in terms of the ordinary component gravitational connection, $\varpi_{a}$, plus the gravitino terms mentioned previously.

\section{B Components of Chiral Superfield Derivatives}

We determine in this Appendix the $\theta=0$ components of the higher spinor derivatives of chiral superfields, as they appear in the component action of subsection 4.1. The 
procedure is straightforward. For example, we write

$$
\begin{aligned}
& \nabla_{+} \bar{\nabla}^{2} \bar{\Phi}\left|=\left[\nabla_{+}, \bar{\nabla}^{2}\right] \bar{\Phi}\right|=i \nabla_{\ddagger} \nabla_{\dot{ }} \bar{\Phi} \mid \\
& =i\left(\mathbf{D}_{\ddagger}+\psi_{\ddagger}^{\alpha} \nabla_{\alpha}+\psi_{\ddagger}^{\dot{\alpha}} \nabla_{\dot{\alpha}}\right) \nabla_{\dot{.}} \bar{\Phi} \mid \\
& =i \mathbf{D}_{\ddagger} \nabla_{\dot{.}} \bar{\Phi}-\psi_{\ddagger}^{-} \nabla_{\ddagger} \bar{\Phi}+i \psi_{\ddagger}^{\dot{+}} \nabla_{\dot{+}} \nabla_{\dot{.}} \bar{\Phi} \mid
\end{aligned}
$$

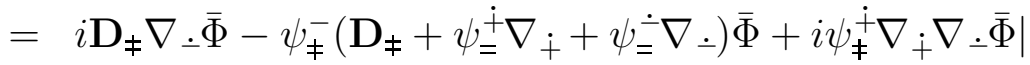

$$
\begin{aligned}
& =i \mathbf{D}_{\ddagger} \psi_{\dot{-}}-\psi_{\ddagger}^{-} \mathbf{D}_{=} \bar{\phi}-\psi_{\ddagger}^{-} \psi_{=}^{\dot{+}} \psi_{\dot{+}}-\psi_{\ddagger}^{-} \psi_{=}^{\dot{\dot{亠}}} \psi_{\dot{-}}+\psi_{\ddagger}^{\dot{+}} \bar{F}
\end{aligned}
$$

where we have used the definition of components of covariant derivatives, as discussed in Appendix A, and the definition of components of $\Phi$.

In a similar manner we find

$$
\nabla_{-} \bar{\nabla}^{2} \bar{\Phi} \mid=-i \mathbf{D}_{=} \psi_{\dot{+}}-\psi_{=}^{+} \mathbf{D}_{\ddagger} \bar{\phi}+\psi_{=}^{+} \psi_{\ddagger}^{\dot{\dot{\phi}}} \psi \cdot+\psi_{=}^{+} \psi_{\ddagger}^{\dot{+}} \psi_{\dot{+}}+\psi_{=}^{\dot{\bar{F}}} \bar{F} .
$$

Next, using the commutation relations and the definition of the components of $R$, we write

$$
\begin{aligned}
& \nabla^{2} \bar{\nabla}^{2} \bar{\Phi}\left|=\nabla_{\ddagger} \nabla_{=} \bar{\Phi}\right|+\frac{1}{2}\left(\nabla_{\dot{+}} \bar{R}\right) \nabla_{\dot{\prime}} \bar{\Phi}\left|+\frac{1}{2} \bar{R} \bar{\nabla}^{2} \bar{\Phi}\right| \\
& =\nabla_{\ddagger} \nabla_{=} \bar{\Phi}\left|+\frac{1}{2} \bar{R} \bar{\nabla}^{2} \bar{\Phi}\right|
\end{aligned}
$$

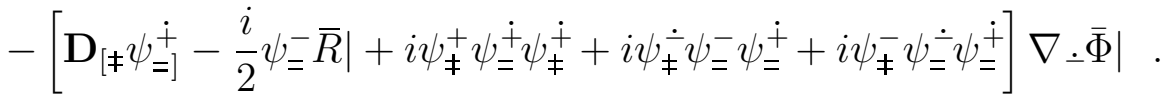

Finally,

$$
\begin{aligned}
& \nabla_{\ddagger} \nabla_{=} \bar{\Phi}\left|=\left(\mathbf{D}_{\ddagger}+\psi_{\ddagger}^{\alpha} \nabla_{\alpha}+\psi_{\ddagger}^{\dot{\alpha}} \nabla_{\dot{\alpha}}\right) \nabla_{=} \bar{\Phi}\right| \\
& =\mathbf{D}_{\ddagger}\left(\mathbf{D}_{=}+\psi_{=}^{\dot{\alpha}} \nabla_{\dot{\alpha}}\right) \bar{\Phi}\left|-\frac{i}{2} \psi_{\neq}^{+} \bar{R} \nabla_{\dot{\Phi}} \bar{\Phi}\right| \\
& +\psi_{\ddagger}^{\dot{+}}\left[\mathbf{D}_{=} \nabla_{\dot{+}}+i \psi_{=}^{+}\left(\mathbf{D}_{\ddagger}+\psi_{\ddagger}^{\dot{\alpha}} \nabla_{\dot{\alpha}}\right)-\psi_{=}^{\dot{-}} \bar{\nabla}^{2}\right] \bar{\Phi} \mid \\
& +\psi_{\ddagger}^{\dot{\dot{*}}}\left[\mathbf{D}_{=} \nabla \dot{-}+i \psi_{=}^{-}\left(\mathbf{D}_{=}+\psi_{=}^{\dot{\alpha}} \nabla_{\dot{\alpha}}\right)+\psi_{=}^{\dot{+}} \bar{\nabla}^{2}\right] \bar{\Phi} \mid \\
& =\mathbf{D}_{\ddagger} \mathbf{D}_{=} \bar{\phi}+\mathbf{D}_{\ddagger} \psi_{=}^{\dot{+}} \psi_{\dot{+}}+\mathbf{D}_{\ddagger} \psi_{=}^{\dot{\dot{亠}}} \psi_{\dot{ }}+\psi_{\ddagger}^{\dot{+}} \mathbf{D}_{=} \psi_{\dot{+}}+\psi_{\ddagger}^{\dot{\dot{\dagger}}} \mathbf{D}_{=} \psi \text {. } \\
& -\frac{i}{2} \psi_{\ddagger}^{+} \bar{B} \psi_{\dot{-}}+i \psi_{\ddagger}^{\dot{+}} \psi_{=}^{+} \mathbf{D}_{\ddagger} \bar{\phi}+i \psi_{\ddagger}^{\dot{\dot{\phi}}} \psi_{=}^{-} \mathbf{D}_{=} \bar{\phi}
\end{aligned}
$$

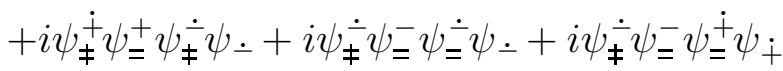

$$
\begin{aligned}
& -i \psi_{\ddagger}^{\dot{\dot{\dot{f}}}} \psi_{=}^{\dot{+}} \bar{F}+i \psi_{\ddagger}^{\dot{+}} \psi_{=}^{\dot{\bar{F}}} \bar{F} \text {. }
\end{aligned}
$$

We now have all the ingredients for computing the component action for the chiral multiplet, and in particular the terms involving the auxiliary fields which are needed for the computation in subsection 4.1. Note that the component covariant derivative appearing above still contains the connection involving gravitino bilinears and a $U(1)$ gauge field. 


\section{Derivation of Twisted Chiral Projection For- mula}

In subsection 4.2 we have presented the projection formula for the component action in terms of a twisted chiral projector. The manipulations that lead to (4.14) are rather baroque, and we can only try to gently guide the enterprising reader (or, more likely, the hapless graduate student) through some of the steps.

We consider the right hand side of (4.11) and study separately the various terms after applying the identity to an arbitrary superspace lagrangian, integrating with $\int d^{2} x e^{-1}$ and evaluating at $\theta=0$.

We begin by considering the term

$$
\begin{aligned}
& \frac{1}{4}\left(\nabla_{-} R\right) \nabla_{+} \mathcal{L} \mid
\end{aligned}
$$

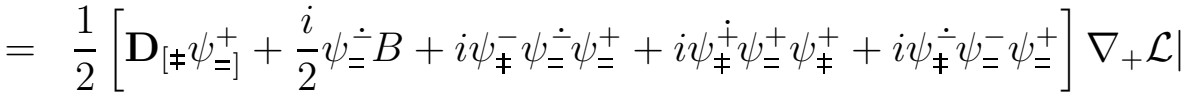

where we have substituted the $\theta=0$ expression for $\nabla_{-} R$. Now,

$$
\begin{aligned}
\mathbf{D}_{\ddagger} \psi_{=}^{+} & =\left(e_{\ddagger}+\omega_{\ddagger} M+\gamma_{\ddagger} \bar{M}\right) \psi_{=}^{+} \\
& =\left(\mathcal{D}_{\ddagger}+\frac{i}{2} A_{\ddagger}^{\prime}\right) \psi_{=}^{+}-\frac{3 i}{2}\left(\psi_{\mp}^{-} \psi_{=}^{\dot{\bar{\nu}}}+\psi_{\ddagger}^{\dot{\bar{q}}} \psi_{=}^{-}\right) \psi_{=}^{+}
\end{aligned}
$$

and

$$
\begin{aligned}
\mathbf{D}_{=} \psi_{\ddagger}^{+} & =\left(e_{=}+\omega_{=} M+\gamma_{\ddagger} \bar{M}\right) \psi_{\neq}^{+} \\
& =\left(\mathcal{D}_{=}+\frac{i}{2} A_{=}^{\prime}\right) \psi_{\neq}^{+}+\frac{i}{2} \psi_{\neq}^{\dot{+}} \psi_{=}^{+} \psi_{\neq}^{+}
\end{aligned}
$$

where we have used the expressions for the connections in (A.24), A.25) and the definition of the gravitational covariant derivative in (A.6). The term above becomes

$$
\begin{aligned}
\frac{1}{4}\left(\nabla_{-} R\right) \nabla_{+} \mathcal{L} \mid= & \frac{1}{2}\left[\mathcal{D}_{[\mp} \psi_{=]}^{+}+\frac{i}{2} A_{[\ddagger}^{\prime} \psi_{=]}^{+}+\frac{i}{2} \psi_{=}^{\dot{亠}} B\right. \\
& \left.-\frac{i}{2} \psi_{\ddagger}^{-} \psi_{=}^{\dot{\dot{ }}} \psi_{=}^{+}+\frac{i}{2} \psi_{\ddagger}^{\dot{+}} \psi_{=}^{+} \psi_{\ddagger}^{+}-\frac{i}{2} \psi_{\ddagger}^{\dot{\dot{亠}}} \psi_{=}^{-} \psi_{=}^{+}\right] \nabla_{+} \mathcal{L} \mid
\end{aligned}
$$

Furthermore, since we are inside a $d^{2} x e^{-1}$ integral, the ordinary gravitational derivative $\mathcal{D}$ can be integrated by parts.

The other terms of a similar type are manipulated in the same fashion.

We consider now

$$
\frac{1}{2} \nabla_{\ddagger} \nabla_{=} \mathcal{L}\left|=\frac{1}{2}\left(\mathbf{D}_{\ddagger}+\psi_{\ddagger}^{\alpha} \nabla_{\alpha}+\psi_{\ddagger}^{\dot{\alpha}} \nabla_{\dot{\alpha}}\right) \nabla_{=} \mathcal{L}\right|
$$


and write out

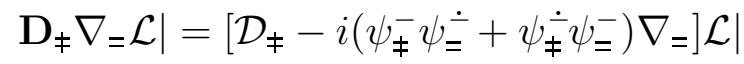

(note that $\nabla_{=} \mathcal{L}$ is $U_{A}(1)$ neutral). Inside the $d^{2} x e^{-1}$ integral, the term $\mathcal{D}_{\ddagger} \nabla_{=} \mathcal{L}$ is a total derivative and can be dropped. For the remaining terms in the above expression we commute the $\nabla_{=}$operator to the left of the $\nabla_{\alpha}$ operators, using the commutation relations in (A.11), so that

$$
\begin{aligned}
& \left(\psi_{\ddagger}^{\alpha} \nabla_{\alpha}+\psi_{\ddagger}^{\dot{\alpha}} \nabla_{\dot{\alpha}}\right) \nabla_{=} \mathcal{L} \mid \\
& =\left(\psi_{\ddagger}^{\alpha} \nabla_{=} \nabla_{\alpha}+\psi_{\ddagger}^{\dot{\alpha}} \nabla_{=} \nabla_{\dot{\alpha}}-\frac{i}{2} \psi_{\ddagger}^{+} \bar{R} \nabla_{\dot{-}}+\frac{i}{2} \psi_{\ddagger}^{\dot{+}} R \nabla_{-}\right) \mathcal{L} \mid
\end{aligned}
$$

We treat the $\nabla_{=} \nabla_{\ddagger} \mathcal{L} \mid$ term in the same fashion.

Assembling everything, the right-hand-side of the original identity becomes

$$
\begin{aligned}
& \frac{1}{2}\left(-R \nabla^{2}+\bar{R} \bar{\nabla}^{2}\right) \mathcal{L} \mid \\
& +\frac{1}{2}\left[\psi_{=}^{+}\left(\nabla_{\ddagger}-\mathcal{D}_{\ddagger}+\frac{i}{2} A_{\ddagger}^{\prime}\right)-\psi_{\ddagger}^{+}\left(\nabla_{=}-\mathcal{D}_{=}+\frac{i}{2} A_{=}^{\prime}\right)+2 \psi_{\ddagger}^{+} \nabla_{=}\right] \nabla_{+} \mathcal{L} \mid \\
& +\frac{1}{2}\left[-\frac{i}{2} \psi_{\ddagger}^{-} \psi_{=}^{\dot{\bar{\psi}}} \psi_{=}^{+}+\frac{i}{2} \psi_{\ddagger}^{\dot{+}} \psi_{=}^{+} \psi_{\ddagger}^{+}-\frac{i}{2} \psi_{\ddagger}^{\dot{\dot{\nu}}} \psi_{=}^{-} \psi_{=}^{+}\right] \nabla_{+} \mathcal{L} \mid \\
& +\left(\text { similar terms with } \nabla_{-} \mathcal{L} \text { etc. }\right)
\end{aligned}
$$

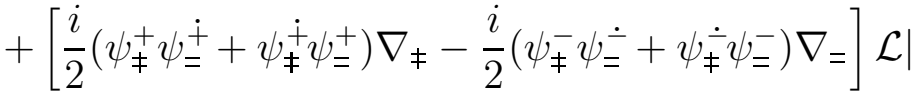

All the terms of the form $B \nabla_{\alpha} \mathcal{L}$ have cancelled.

Next we work out

$$
\begin{aligned}
\frac{1}{2} \nabla_{\ddagger} \nabla_{+} \mathcal{L} \mid & =\frac{1}{2}\left(\mathbf{D}_{\ddagger}+\psi_{\ddagger}^{\alpha} \nabla_{\alpha}+\psi_{\ddagger}^{\dot{\alpha}} \nabla_{\dot{\alpha}}\right) \nabla_{+} \mathcal{L} \mid \\
& =\frac{1}{2}\left[\mathcal{D}_{\ddagger}-\frac{i}{2} A_{\ddagger}^{\prime}+\frac{i}{2}\left(\psi_{\ddagger}^{-} \psi_{=}^{\dot{\dot{\prime}}}+\psi_{\ddagger}^{\dot{\dot{\dot{y}}}} \psi_{=}^{-}\right)+\psi_{\ddagger}^{\alpha} \nabla_{\alpha}+\psi_{\ddagger}^{\dot{\alpha}} \nabla_{\dot{\alpha}}\right] \nabla_{+} \mathcal{L} \mid
\end{aligned}
$$

and, in a similar manner, $\frac{1}{2} \nabla_{=} \nabla_{+} \mathcal{L} \mid$.

Substituting into (C.8), we find that all three-gravitino terms cancel, and the two-gravitino terms in the last line are also cancelled.

We obtain, finally, for the right-hand-side of the identity (4.11)

$$
\begin{aligned}
& \left\{\left[\psi_{\ddagger}^{+} \psi_{=}^{-}+\psi_{\ddagger}^{-} \psi_{=}^{+}-\frac{1}{2} R\right] \nabla^{2}+\left[\psi_{\ddagger}^{\dot{+}} \psi_{=}^{\dot{\bar{\Xi}}}+\psi_{\ddagger}^{\dot{\dot{\bar{y}}}} \psi_{=}^{\dot{+}}+\frac{1}{2} \bar{R}\right] \nabla^{2}\right. \\
& +\left(\psi_{\ddagger}^{+} \psi_{=}^{\dot{\dot{ }}}+\psi_{\ddagger}^{\dot{\dot{亠}}} \psi_{=}^{+}\right) \nabla_{+} \nabla_{\dot{\prime}}+\left(\psi_{\ddagger}^{\dot{+}} \psi_{=}^{-}+\psi_{\ddagger}^{-} \psi_{=}^{\dot{+}}\right) \nabla_{\dot{+}} \nabla_{-} \\
& \left.+\psi_{\ddagger}^{+} \nabla_{=} \nabla_{+}+\psi_{=}^{-} \nabla_{\ddagger} \nabla_{-}+\psi_{\ddagger}^{\dot{+}} \nabla_{=} \nabla_{\dot{+}}+\psi_{=}^{\dot{\dot{\prime}}} \nabla_{\ddagger} \nabla_{\dot{-}}\right\} \mathcal{L} \mid
\end{aligned}
$$

which can be rearranged into the form given in the main text. 


\section{References}

[1] M.T. Grisaru and M.E. Wehlau, Int. J. Mod. Phys. A10 (1995) 753.

[2] M.T. Grisaru and M.E. Wehlau, (2,2) Supergravity in the light-cone gauge, hep-th/9505068, Nucl. Phys. B, to be published.

[3] S.J. Gates, Jr., M.T. Grisaru and M.E. Wehlau, A study of general 2D, N=2 supergravity coupled to matter in superspace, in preparation.

[4] S. J. Gates, Jr., M.T. Grisaru, M. Roček, and W. Siegel, Superspace, (BenjaminCummings, 1983).

[5] I.L. Buchbinder and S.M. Kuzenko, Ideas and Methods of Supersymmetry and Supergravity, (Institute of Physics, 1995). 